\title{
COVID-19 Pandemic with Human Mobility Across Countries
}

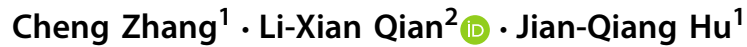

Received: 20 May 2020 / Revised: 27 June 2020 / Accepted: 6 July 2020 / Published online: 3 August 2020 (c) Operations Research Society of China, Periodicals Agency of Shanghai University, Science Press, and Springer-Verlag GmbH Germany, part of Springer Nature 2020

\begin{abstract}
This study develops a holistic view of the novel coronavirus (COVID-19) spread worldwide through a spatial-temporal model with network dynamics. By using a unique human mobility dataset containing 547166 flights with a total capacity of 101455913 passengers from January 22 to April 24, 2020, we analyze the epidemic correlations across 22 countries in six continents and particularly the changes in such correlations before and after implementing the international travel restriction policies targeting different countries. Results show that policymakers should move away from the previous practices that focus only on restricting hotspot areas with high infection rates. Instead, they should develop a new holistic view of global human mobility to impose the international movement restriction. The study further highlights potential correlations between international human mobility and focal countries' epidemic situations in the global network of COVID-19 pandemic.
\end{abstract}

Keywords COVID-19 · Pandemic $\cdot$ Human mobility · International correlation · International travel restriction

Mathematics Subject Classification 91C15 -90B10 · 91B72 · 92D30

This work was supported by the National Natural Science Foundation of China (Nos. 91846302 , 71720107003, and 71973107).

Electronic supplementary material The online version of this article (https://doi.org/10.1007/s40305-02 0-00317-6) contains supplementary material, which is available to authorized users.

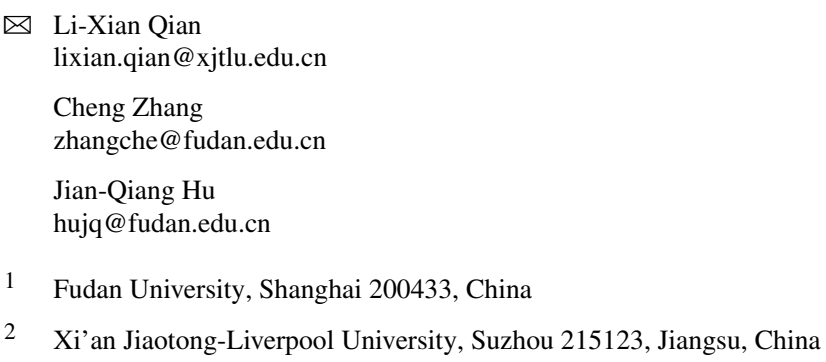




\section{Introduction}

Although countries worldwide have gradually restricted nonessential international travel, particularly those from high-infection areas, it does not seem to have had the desired effect of stopping the spread of the novel coronavirus (COVID-19) in the past few weeks. The epidemic continues to be severe in the European and North American regions. It has even gradually spread to more areas, such as Africa and South America, leading to controversy over the pathway of the epidemic transmission $[1,2]$. The urgent situation needs a better assessment of the COVID-19 spread under global human mobility [3, 4].

Besides non-pharmaceutical interventions within each country [5], restrictions on nonessential international travel from epidemic areas are proposed as a critical strategy to slow the spread [6]. It intends to cut off people's outbound movement from a country when it becomes a hot spot of the epidemic. This approach may not be as effective as policymakers expect if they ignore the current reality of global human mobility. Another important decision-making factor that has been overlooked is how to impose internal movement control policies, such as curfew or other forms of travel restriction, in the infected areas. These issues are particularly important in designing a global strategy to respond to the COVID-19 dynamics and to recover social and economic activities.

Figure 1 summarizes three different views on understanding the role of human mobility in the pandemic. The first view is a focal view that addresses the role of internal mobility and movement controls in tackling epidemic in a single country while ignoring the influence of inbound and outbound mobility [7-10]. The second one is a dyadic view that explores the role of international mobility and travel restriction between a centric country (usually a hotspot area in the epidemic) and some other countries [11-13]. This approach usually does not take into account the impact of population movements other than those of the centric country, and in addition, only the one-way impact of the centric country on other countries. The third one is a holistic view that simultaneously analyzes the multiple paths of international mobility and travel restrictions in a global network and thus allows for the correlation of each path in the network. In this way, the networked approach allows the study of simultaneous and asymmetric effects between multiple countries through different mobility pathways.

Global network-based analysis is now more urgently needed than the previous focal view approach [7-10] or the dyadic view approach [11-13], that is, investigating epidemic developments between countries in the global network where each country is a node and the inter-country human mobility between two countries is represented by an edge. In this way, scholars and policymakers can gain the holistic insights on the virus' spread across the globe and develop effective and worldwide-coordinated measures to contain the pandemic [14].

This study responds to this urgent call by developing a spatial-temporal model with network dynamics $[15,16]$ to understand the correlation between the COVID19 epidemics in different countries accounting for the inter-country human mobility and international travel restrictions targeting different countries. Our analysis of the COVID-19 pandemic starts from January 22, 2020, when the epidemic was officially reported by the World Health Organization (WHO), to April 24, 2020, when the number 


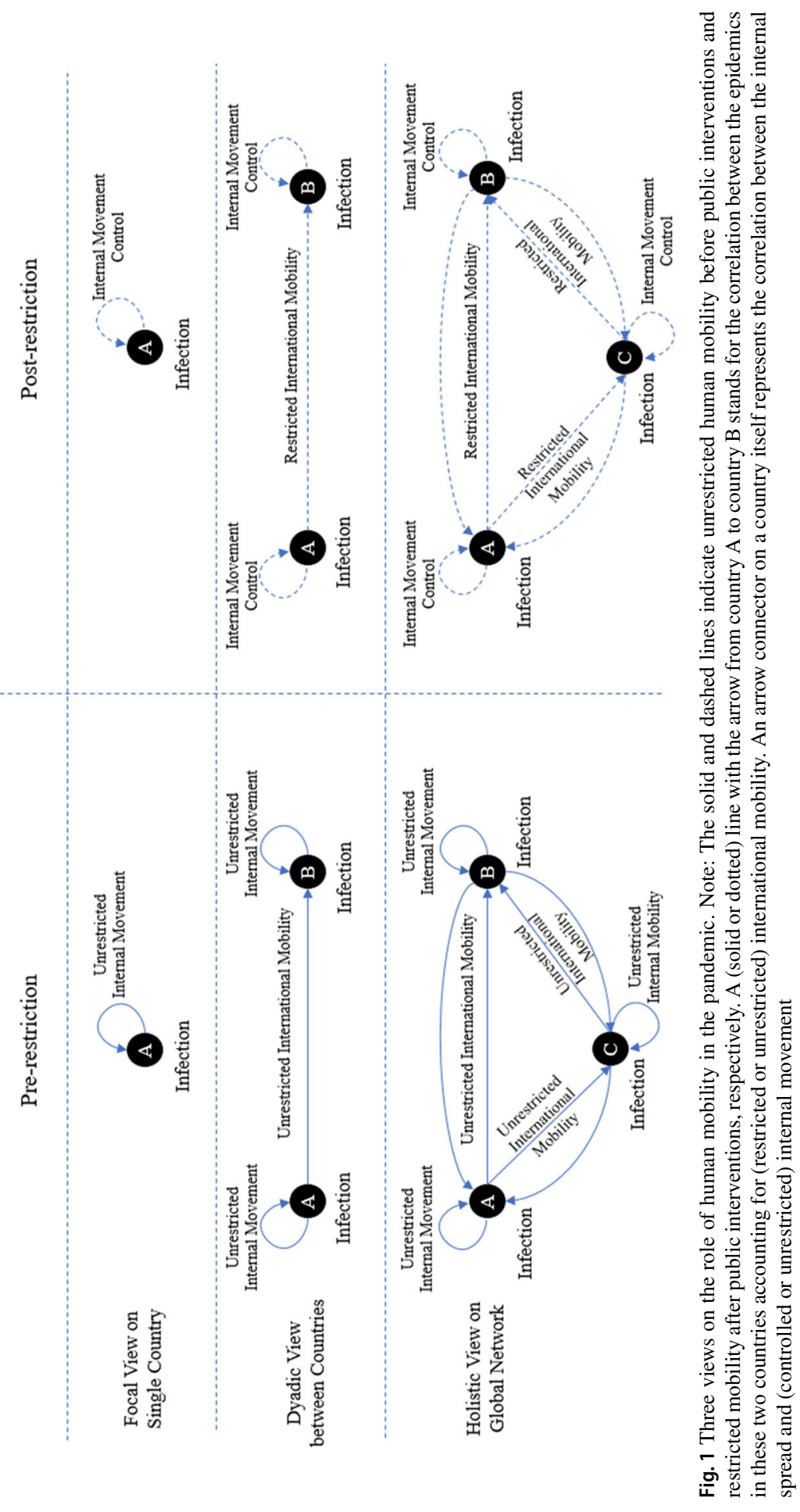


of COVID-19 infections exceeded 2.6 million worldwide. Based on the timing of the outbreak and the scale in terms of the accumulated confirmed infection numbers in the COVID-19 pandemic, we selected 22 countries from six continents, which accounted for $86.70 \%$ of total infection amount worldwide as of April 24, 2020. Detailed information of the countries is provided in Table S1 in supplementary materials.

We collected the daily number of confirmed COVID-19 infections in every country from the WHO. We also use a unique dataset containing 547166 flights with a total capacity of 101455913 passengers among the 22 countries from January 22 to April 24,2020 . In the follow-up analyses, the information is aggregated to the countryday level, resulting in 23885 daily pairs of international travel movement across six continents. Figure S1 illustrates a clear decreasing trend since early March regarding the capacity and number of international flights among these 22 countries. We also compile data from multiple sources containing international travel restriction and internal movement control policies (such as curfew and other forms of domestic travel restriction), as summarized in Table $\mathrm{S} 1$.

By treating each country as a node in the global network and the international human mobility as the connections between countries, we model the dynamic process of the epidemic. The unit of analysis in this study is the number of confirmed infections in country $i$ on day $t$. At the beginning of the epidemic, every country had zero infection and human mobility was unrestricted between countries. Likewise, there were neither non-pharmaceutical interventions within countries nor international mobility restriction between countries. Therefore, countries around the world had formed a fully connected human mobility network.

During the pandemic period, however, travel restrictions were introduced by countries gradually and applied to travelers from different countries at different time points, which led to a dynamically changing global human mobility network. The restriction reduces the human mobility between countries. If the action, saying the travel restriction from a hotspot country A to another country B, was introduced in time and did take effect, we would expect to see a weaker association between the epidemic in country $\mathrm{A}$ and the daily new cases in country B in the controlled international travel period than in the pre-controlled period, which is equivalent to a positive effect of such correlation before the introduction of international travel restriction with reference in the restricted period. This would effectively imply a decreasing association of epidemic situations from country A to country B with the introduction of international travel restriction, largely due to the fewer number of travelers from country A to country B. However, if the international travel restriction is not effectively implemented by country B regarding timing and targeting countries, we might even observe a stronger association between the daily new cases in country B and the epidemic in country A after country B bans the entry of international travelers from country A, which is equivalent to a negative association between the daily increment in country B and the epidemic in country $\mathrm{A}$ in the pre-controlled period for international travel with reference in the controlled period.

In addition, reducing the spread of the epidemic does not solely depend on the international travel restriction and would also rely on the effective internal movement control within each country. Specifically, even if country B reduces international movement from the hotspot country A, the virus may still have the chance to spread within 
country B (e.g., via internal spread or from the third country), so that the impact of the epidemic in country B cannot be reduced without effective internal movement control policies. Therefore, if country B effectively implements internal movement control, we would also expect the control to work with international mobility restriction and observe a decreasing epidemic trend in country B, after a certain lagged period.

This idea is applied to all the countries in the network, taking into account the multiple paths of international mobility and travel restrictions between any two of them simultaneously. Through such a global network perspective, we aim to examine the correlations of COVID-19 epidemics in respective countries and the extent to which the human mobility and the introduction of international travel restriction targeting hotspot countries influence the pandemic.

\section{Data and Model}

\subsection{Data}

We collected multi-sourced datasets in this study. The daily numbers of confirmed infections of all countries were collected from the WHO COVID-19 Dashboard. ${ }^{1}$ Based on the timing of the outbreak occurred and the scale in terms of their accumulated confirmed infection numbers during the outbreak, we selected 22 countries from six continents: 6 countries from Asia (China, Iran, India, Japan, South Korea, and Turkey), 10 countries from Europa (Belgium, France, Germany, Italy, Netherlands, Portugal, Russian Federation, UK, Spain, and Switzerland), 2 countries from North America (Canada and USA), 2 countries from South America (Brazil, Ecuador), 1 country from Africa (South Africa), and 1 country from Oceania (Australia). These 22 countries accounted for $86.70 \%$ of the total infection amount worldwide as of April 24, 2020. In addition to the WHO data, we also collected data from and the COVID-19 Data Repository by the Center for Systems Science and Engineering (CSSE) at Johns Hopkins University [17] to replicate the analysis.

We further use a unique human mobility dataset containing daily global commercial flights among the selected 22 countries between January 22, 2020, and April 24, 2020, from a leading data consulting company in the civil aviation industry, VariFlight. ${ }^{2}$ The dataset contains information on the origin country, destination country, date, the number of flights, and total capacity in terms of the maximum available seats. In summary, this dataset provides information on 547166 flights between 23855 pairs of origin and destination countries that covers a total capacity of 101455913 passengers during the period.

Moreover, we collected data on the international travel restriction between countries and the internal movement control within every country from Oxford COVID-19 Government Response Tracker $^{3}$ and GardaWorld Crisis24 Global Portal, ${ }^{4}$ which timely

\footnotetext{
1 WHO COVID-19 Dashboard (https://covid19.who.int/).

2 http://www.variflight.com/en/.

3 https://www.bsg.ox.ac.uk/research/publications/variation-government-responses-covid-19.

4 https://www.garda.com/crisis 24.
} 
document epidemic prevention policies in countries around the world. In a global network, the path connection from country A to country B is set by value 1 at day 0 , representing the availability of international movement from A to B. Such connection is disabled on day $t$, due to either the internal movement control in country A or the entry ban for travelers from country A into B. Thus, on day $t$ onward, the connection from A to B is set to be 0 to represent such disconnection. Table S1 summarizes the timing and scale of the outbreak, as well as travel restriction policies by the 22 countries.

\subsection{Dynamic Spatial Network Model}

The unit of our analysis is the number of confirmed infections in country $i$ on day $t$. Given the spatial nature of this research, we develop a dynamic network model based on the spatial-temporal features $[15,16]$ to examine the extent to which the number of newly confirmed infections in each country is correlated with the cumulative number of infections in each of the remaining 21 countries, by taking into account (1) the mobility volume between countries, (2) the introduction of inter-country travel restriction policies targeting different countries at different time point, and (3) internal movement restriction within each country. Specifically, the number of newly confirmed infections in the country $i(i=1$ to 22$)$ on day $t(t=1$ to 94 , starting at January 22 till April 24), $\mathrm{NCI}_{i, t}$, is given by

$$
\mathrm{NCI}_{i t}=\alpha_{1 i} \mathrm{NCI}_{i, t-\tau}+\alpha_{2 i} \operatorname{PreIR}_{i, t-\omega}+\alpha_{3 i j} \operatorname{FCap}_{i j, t-\theta}^{\prime} \boldsymbol{W}_{t-\theta}^{\prime} \overline{\boldsymbol{C I}} \boldsymbol{I}_{j, t-\theta}+\varepsilon_{i t},
$$

where $\varepsilon_{i t}$ is the error term, the coefficient $\alpha_{1 i}$ measures the $\tau$-day lagged effect of new infections in the same country $\left(\mathrm{NCI}_{i, t-\tau}\right)$, and $\alpha_{2 i}$ captures the $\omega$-day lagged effect related to internal movement restriction in the same country (PreIR $\left.\operatorname{IR}_{i, t-\omega}\right)$, such as social distancing and home-stay orders, where

$$
\operatorname{PreIR}_{i, t-\omega}=\left\{\begin{array}{l}
1, \text { if internal movement is fully allowed in country } i \text { on day } t-\omega, \\
0, \text { otherwise. }
\end{array}\right.
$$

Specifically, the significantly positive value of $\alpha_{2 i}$ represents the positive association between fully allowed internal movement (with reference to the restriction) and the new infections in the same country $i$ after $\omega$ days. This effectively means that there was the less severe epidemic in the controlled period $\left(\operatorname{PreIR}_{i, t-\omega}=0\right)$ than in the pre-controlled period for internal movement $\left(\operatorname{PreIR}_{i, t-\omega}=1\right)$ and thus implies the effectiveness of internal movement control policy in country $i$. On contrary, the significantly negative value of $\alpha_{2 i}$ would suggest the more severe epidemic in the controlled period (PreIR ${ }_{i, t-\omega}=0$ ) than in the pre-controlled period (PreIR $\operatorname{IR}_{i, t-\omega}=1$ ), while the insignificant value of $\alpha_{2 i}$ means there is no statistical difference on the epidemic in country $i$ before and after introducing the internal movement control policy.

Furthermore, $\overline{\boldsymbol{C I}}$ it is the number of cumulative confirmed infections in each of the rest of countries other than country $i$ by day $t(j \neq i)$ and FCap ${ }_{i j t}^{\prime}$ stands for the human mobility from country $j$ to $i$, such as the number of direct flights or maximum flight seats in the direct flights from country $j$ to $i$ on date $t$, which might be further 
restricted by the international travel control policies introduced by country $i$ targeting different countries on different days. To capture such dynamic international travel controls, $\boldsymbol{W}_{t}^{\prime}$ is the time-dependent spatial weight matrix of the international mobility network between all considered countries on day $t$, with its element $w_{i j t}$ defined as

$$
w_{i j t}=\left\{\begin{array}{l}
1, \text { if regular travel from country } j \text { to } i \text { is allowed on date } t \\
0, \text { otherwise. }
\end{array}\right.
$$

Specifically, the travel from country $j$ to $i$ on day $t$ might be restricted due to the respective policies in the origin or destination countries. In the origin country $j$, its internal movement restriction on day $t$ might make regular travelers difficult or even impossible to leave country $j$. The destination country $i$ may also ban the entry of travelers from country $j$. Therefore, $\alpha_{3 i j}$ (i.e., the coefficient of the term FCap $\left._{i j, t-\theta}^{\prime} \boldsymbol{W}_{t-\theta}^{\prime} \overline{\boldsymbol{C I}} \boldsymbol{I}_{j, t-\theta}\right)$ measures the $\theta$-day lagged correlation between the epidemic in country $j$ and the daily new cases in country $i$, accounting for international mobility capacity and the international travel restrictions, where the $\theta$-day lag can be largely attributed to the incubation period. The significantly positive value of $\alpha_{3 i j}$ means that the correlation between the cumulative epidemic in country $j$ and the $\theta$ day lagged new infections in country $i$ is stronger in the pre-controlled period for the international mobility $\left(w_{i j t}=1\right)$ than in the international mobility controlled period $\left(w_{i j t}=0\right)$. In other words, the positive value of $\alpha_{3 i j}$ suggests the weaker correlation effect in the international mobility controlled period and thus implies the effectiveness of introducing international mobility control policies on containing the COVID-19 epidemic in country $i$. Conversely, the significantly negative effects of $\alpha_{3 i j}$ means that the stronger correlation between the cumulative epidemic in country $j$ and the $\theta$-day lag new infections in country $i$ in the international mobility controlled period $\left(w_{i j t}=0\right)$ than in the pre-controlled period $\left(w_{i j t}=0\right)$, which might be due to the delayed implementation of international travel restriction or the myopia of only banning international travelers from the existing hotspot but ignoring the other countries with emerging epidemics. In addition, the insignificant value of $\alpha_{3 i j}$ means the intercountry correlation of epidemics remains largely unchanged with the introduction of international travel restrictions from country $j$ to $i$.

\subsection{Estimating the Model}

The econometric model we develop above has a system of 22 simultaneous equations with the time-dependent spatial weight matrix $\boldsymbol{W}_{t}^{\prime}$. This unique feature makes our model differs from the conventional spatial models, including the dynamic spatial panel model, which typically use the time-invariant spatial weight matrix based on the geographical characteristics [16]. Therefore, we follow the process below to estimate the model.

1. Code the elements of spatial weight matrix $\left(w_{i j t}\right)$, which may vary by the origin, destination, and date, based on the international travel information and internal movement control policies, as summarized in Table S1. 
2. Organize the data into a time series format, with each row representing the date and each column for the different data variables on that date, such as the coded elements of the spatial weight matrix, the number of infection cases in every country and the numbers of flights and maximum available seats in every pair of international mobility route.

3. Create time-lagged variables where necessary. In the empirical analysis, we take $\tau=1$ to one-day lagged effect of new infections, $\omega=14$ to check the effect of internal movement restriction within each country, and $\theta=14$ to capture the influence of 14-day incubation period of the COVID-19 on its international spread.

4. Construct an interdependent system of linear regression equations, and use seemingly unrelated regression (SUR) [18-20] to estimate the parameters as specified in Eq. (1). The SUR is also known as joint generalized least squares (JGLS), and it is a generalization of OLS for multi-equation systems by allowing for the correlation of the error term of every equation. In an $m$-equation system for T-period observations,

$$
\left[\begin{array}{c}
y_{1} \\
y_{2} \\
\vdots \\
y_{m}
\end{array}\right]=\left[\begin{array}{cccc}
X_{1} & 0 & \cdots & 0 \\
0 & X_{2} & \cdots & 0 \\
\vdots & \vdots & \ddots & \vdots \\
0 & 0 & \cdots & X_{m}
\end{array}\right]\left[\begin{array}{c}
\beta_{1} \\
\beta_{2} \\
\vdots \\
\beta_{m}
\end{array}\right]+\left[\begin{array}{c}
e_{1} \\
e_{2} \\
\vdots \\
e_{m}
\end{array}\right]=\mathbf{X} \beta+\boldsymbol{e}
$$

The variance-covariance matrix for the error term vector $e$ can be written as

$$
V=\Sigma \otimes I=\left[\begin{array}{cccc}
\sigma_{11} I & \sigma_{12} I & \cdots & \sigma_{1 m} I \\
\sigma_{21} I & \sigma_{22} I & \cdots & \sigma_{2 m} I \\
\vdots & \vdots & \ddots & \vdots \\
\sigma_{m 1} I & \sigma_{m 2} I & \cdots & \sigma_{m m} I
\end{array}\right]
$$

where $\otimes$ is the Kronecker multiplication operator, $\Sigma$ is an $m^{*} m$ positive definite symmetric matrix with $\sigma_{11}$ as the variance of the error in the first equation and $\sigma_{i j}$ as the covariance between the errors of the $i$ th and $j$ th equations, and $I$ is the identity matrix. The estimation process of SUR is as follows:

a. First apply OLS to every equation and obtain the residual of every equation, $e_{i}$ $(i=1,2, \cdots, \mathrm{m})$.

b. Since $\Sigma$ is typically unknown, the elements of $\Sigma$ can then be estimated using

$$
\hat{\sigma}_{i j}=s_{i j}=\frac{e_{i}^{\prime} e_{j}}{T}
$$

c. Flexible generalized least square (FGLS) estimators are used to estimate the coefficients

$$
\hat{\boldsymbol{\beta}}_{\mathrm{FGLS}}=\left[X^{\prime} V^{-1} X\right]^{-1} X^{\prime} V^{-1} y
$$


where $\left[X^{\prime} V^{-1} X\right]^{-1}$ is the variance-covariance matrix of the estimated coefficients.

The model is implemented using the SUR estimator in the SYSLIN procedure in SAS. The SAS code is available in supplementary materials.

\section{Results}

Table 1 presents the estimation results, where the red and blue colors highlight the positive and negative effects, respectively, both significant at 5\% level. Overall, the 14-day lagged internal movement control policy is found to have significant negative effects (i.e., $\alpha_{2 i}<0$ ) in 18 countries, which suggests the epidemics became more severe in these countries in spite of the introduction of movement control policies such as stay-at-home order.

The correlations (and the changes of correlations) between the epidemics across different countries are presented in the $22 * 22$ matrix under the coefficients $\alpha_{3 i j}$ in Table 1. First, the USA, South Africa, Switzerland, the Netherlands, and Brazil are positively correlated (i.e., in red cells) with the epidemics in three or more countries, which means such correlations (with 14-day lag) have been weaker after introducing international travel restriction across these countries. At the same time, the daily new cases in each of these five countries are also negatively correlated (i.e., in blue cells) with the epidemics in at least three other countries, which implies the 14-day lagged epidemic became more severe in these five countries even after introducing the international travel restriction in respective countries. Overall, there were 30 significantly positive inter-country correlations and 29 significantly negative inter-country correlations as presented in Table 1, which indicates that in a global network the effect of international travel restriction on containing COVID-19 pandemic is not as straightforward as normally expected. The full estimation results are summarized in Table S2.

As shown in Table 1, the international travel restriction for the travelers from China is found to have insignificant effect on the changes in the association between the epidemic in China and the outbreaks in the remaining 21 countries. That is, all the associations between China's cumulative infection number and the daily new infection numbers in other 19 countries with direct flights are insignificant (note that China has no direct flights to Brazil and Ecuador). The main reason may be due to China's early and immediate restriction not only on internal movement but also on nonessential international travel outbound from China in late January 2020. What is more, the number of new infections in China is not significantly correlated with the cumulative infections in other countries except Italy $\left(\alpha_{3}^{\text {ITA } \rightarrow \text { CHN }}=0.539, p<0.001\right)$, which means that the correlation between the epidemic in Italy and daily new cases in China has been weaker after restricting foreigners entering China and such correlation between other countries (except Italy) and China has not changed significantly. Other similar cases are South Korea and Iran, in which the inter-country epidemic correlations have not changed significantly before and after the international travel restriction, given the insignificant correlations (1) between their cumulative infection numbers and 


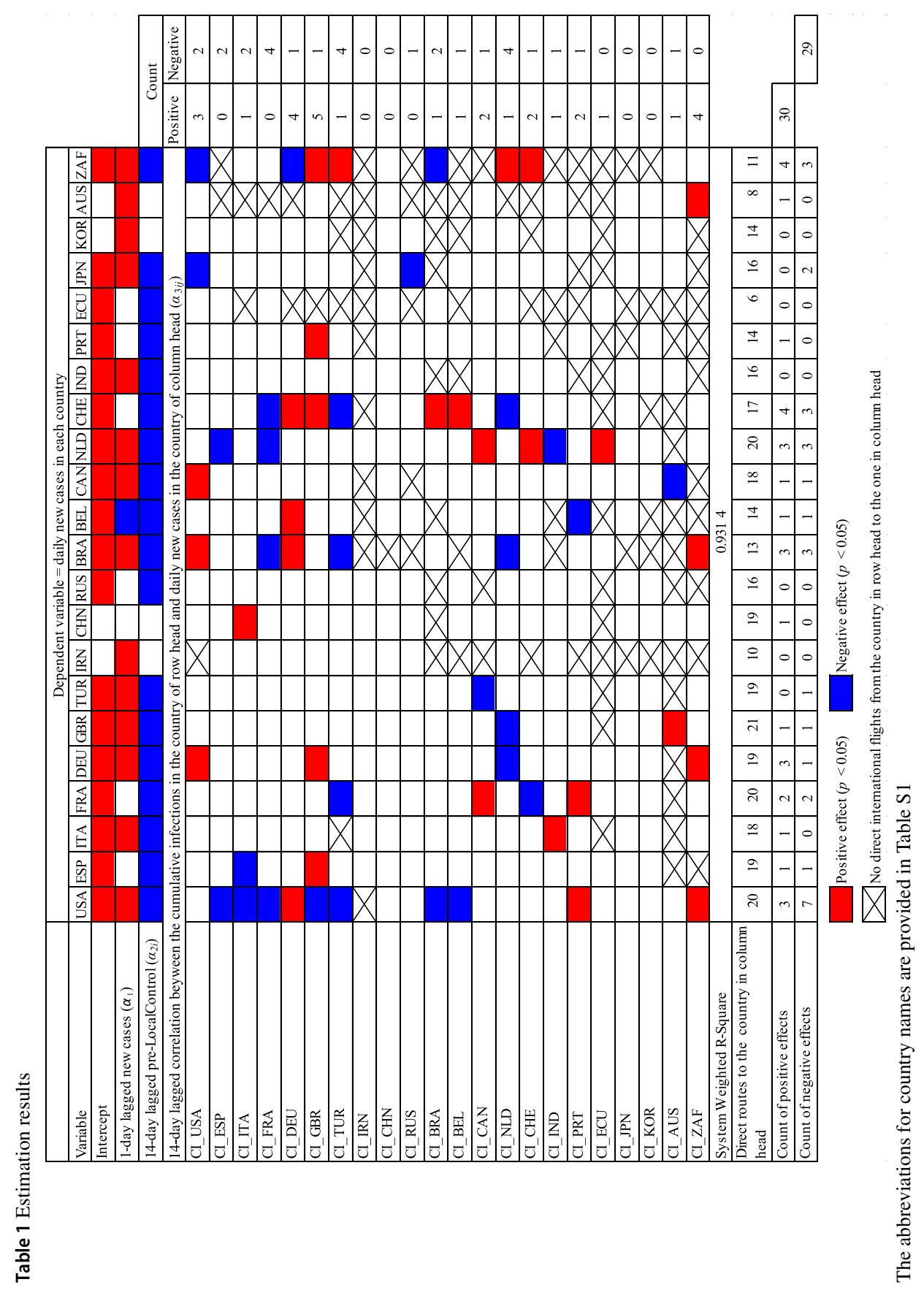


other countries' lagged daily new cases and (2) between other countries cumulative infections and their own lagged daily new cases.

However, other Asian countries such as Japan and India experienced a different situation as they have more changes in their connections with other countries. The correlation between the cumulative cases in Japan and other countries' outbreaks has not changed significantly, but we find negative effects on the correlations between the lagged number of daily new cases in Japan and the cumulative infection numbers in the $\mathrm{USA}\left(\alpha_{3}^{\mathrm{USA} \rightarrow \mathrm{JPN}}=-0.123, p<0.05\right)$ and Russia $\left(\alpha_{3}^{\mathrm{RUS} \rightarrow \mathrm{JPN}}=-0.112, p<0.05\right)$. This means the correlation between these two countries' epidemic and the daily increment in Japan has been stronger, even after the international travel restrictions imposed on American and Russian travelers to visit Japan. Although the outbreak of COVID-19 in India was quite late, its epidemic size (i.e., cumulative number of infections) has a significant association with the numbers of daily new cases in European countries, such as Italy in the pre-controlled period for international travel $\left(\alpha_{3}^{\mathrm{IND} \rightarrow \mathrm{ITA}}=0.310, p\right.$ $<0.05)$ and the Netherlands in the controlled period $\left(\alpha_{3}^{\mathrm{IND} \rightarrow \mathrm{NLD}}=-0.688, p<0.01\right)$.

The results also show that the number of daily new cases in the USA is largely associated with the epidemics in European countries in both the pre-controlled and controlled periods for international travel, as well as in South American and African countries. Specifically, we find that the cumulative infections in Germany, Portugal, and South Africa are positively associated with the new infections in the USA $\left(\alpha_{3}^{\mathrm{DEU} \rightarrow \mathrm{USA}}=2.195, p<0.001 ; \alpha_{3}^{\mathrm{PRT} \rightarrow \mathrm{USA}}=1.091, p<0.01 ; \alpha_{3}^{\mathrm{ZAF} \rightarrow \mathrm{USA}}=0.269\right.$, $p<0.01$, which suggests the inter-country correlations between these countries epidemic and the daily new cases in the USA have been weaker after introducing international travel restrictions to the USA in March 2020. In comparison, even after the USA introduced the restriction for foreigners to enter, its lagged number of new infections is found to have stronger correlation with the cumulative infection sizes in several other countries than in the pre-controlled period, as indicated by the negative correlation coefficients with Spain $\left(\alpha_{3}^{\mathrm{ESP} \rightarrow \mathrm{USA}}=-0.421, p<0.001\right)$, Italy $\left(\alpha_{3}^{\mathrm{ITA} \rightarrow \mathrm{USA}}=-0.051, p<0.05\right)$, France $\left(\alpha_{3}^{\mathrm{FRA} \rightarrow \mathrm{USA}}=-3.277, p<0.001\right)$, the UK $\left(\alpha_{3}^{\mathrm{GBR} \rightarrow \mathrm{USA}}=-0.089, p<0.05\right)$, Belgium $\left(\alpha_{3}^{\mathrm{BEL} \rightarrow \mathrm{USA}}=-0.591, p<0.05\right)$, Brazil $\left(\alpha_{3}^{\mathrm{BRA} \rightarrow \mathrm{USA}}=-0.233, p<0.05\right)$, and Turkey $\left(\alpha_{3}^{\mathrm{TUR} \rightarrow \mathrm{USA}}=-0.078, p<0.01\right)$. Besides, we find three positive and two negative correlations between the cumulative infection number in the USA and the daily new infections of five countries, which are Germany, UK, Canada, South Africa, and Japan. In Canada, another North American country, the daily new cases is found to have stronger associations with the cumulative infection numbers in France and the Netherlands (in the pre-controlled international travel period), as well as Turkey (in the controlled international travel period. In addition, the Canadian cumulative epidemic is found to have stronger correlations with daily new cases in the USA during the pre-controlled period and with the daily increment in Australia after introducing the international travel restriction. Overall, the North American countries have the strong association with different European countries during the different periods of COVID-19 pandemic, followed by those in Africa, South America, and Asia.

We further find a close circle of mutual correlations among European countries. Taking Spain as an example, its number of daily new cases is found to have stronger correlation with the cumulative situations in different European countries, such as 
the UK $\left(\alpha_{3}^{\mathrm{GBR} \rightarrow \mathrm{ESP}}=0.228, p<0.001\right)$ in the pre-controlled period, and Italy $\left(\alpha_{3}^{\text {ITA } \rightarrow \text { ESP }}=-0.142, p<0.05\right)$ in the controlled international travel period. Furthermore, the negative correlations between the epidemic in Spain and the numbers of new cases in the Netherlands and the USA indicate such association becomes stronger even after the international travel has been restricted from Spain to these two countries. Similarly, the number of new cases in Germany is significantly associated with the epidemics in the UK and the Netherlands as well as the USA and South Africa. Meanwhile, the epidemic in Germany also has strong correlations with the daily increment in Belgium, Switzerland, the USA, Brazil, and South Africa. Importantly, the correlations related to Germany are mostly positive, except with the Netherlands and South Africa, which implies most associations became weaker after imposing international travel restriction to/from Germany.

Our analysis also reveals the third-wave epidemics differ in West Asia (e.g., Turkey), East Europe (e.g., Russia), South America (e.g., Brazil and Ecuador), and Africa (e.g., South Africa). Specifically, the correlation between the lagged number of daily new cases in Turkey and the Canadian cumulative epidemic size became stronger in the controlled international mobility period than in the pre-controlled period $\left(\alpha_{3}^{\mathrm{CAN} \rightarrow \mathrm{TUR}}=-0.092, p<0.01\right)$; the Turkish correlation between its cumulative infection number and the daily increment of South Africa was weaker with the international travel restriction, but its correlation with the daily increments in France, Switzerland, USA, and Brazil is stronger in the controlled international travel period than in the pre-controlled period. The cumulative epidemic in Russia is significantly associated with the recent upsurge of the COVID-19 new cases in Japan $\left(\alpha_{3}^{\mathrm{RUS} \rightarrow \mathrm{JPN}}=-0.112, p<0.05\right)$. In comparison, the number of daily new cases in Brazil is strongly correlated with the cumulative epidemics in the USA, Germany, and South Africa in the pre-controlled international travel period, and with the Netherlands, France, and Turkey in the controlled period. In addition, the cumulative infection in South Africa has stronger correlation with the daily increment in the USA in the precontrolled period, but the daily new cases in South Africa are more correlated with the epidemic size in the USA in the controlled period. In addition, the daily increment in South Africa is more correlated with the cumulative infection numbers in Germany and Brazil in the controlled period, and with that of UK, the Netherlands, Switzerland, and Turkey in the pre-controlled period.

In summary, our analysis provides insightful findings on the changes in correlations of the COVID-19 pandemic across countries with the introduction of international travel restrictions, by taking into account the international human mobility. As illustrated in Fig. 2, the size of each node represents the total infection numbers in the corresponding country by April 24, 2020. A red (blue) line represents the positive (negative) correlation between each pair of countries with the strength of the line representing the absolute value of the standardized correlation coefficient.

To check the robustness of our results, we repeat our analysis by using the number of international flights instead of flight capacity for international mobility volume (i.e., $\mathrm{FCap}_{i j t}^{\prime}$ ) in the model. The results are consistent with that in the main analysis and summarized in Table S3. We further consider the situation without flight information available but keeping the time-dependent spatial weight matrix $\left(\boldsymbol{W}_{t}^{\prime}\right)$ to indicate whether a country implemented international travel restriction with another country 


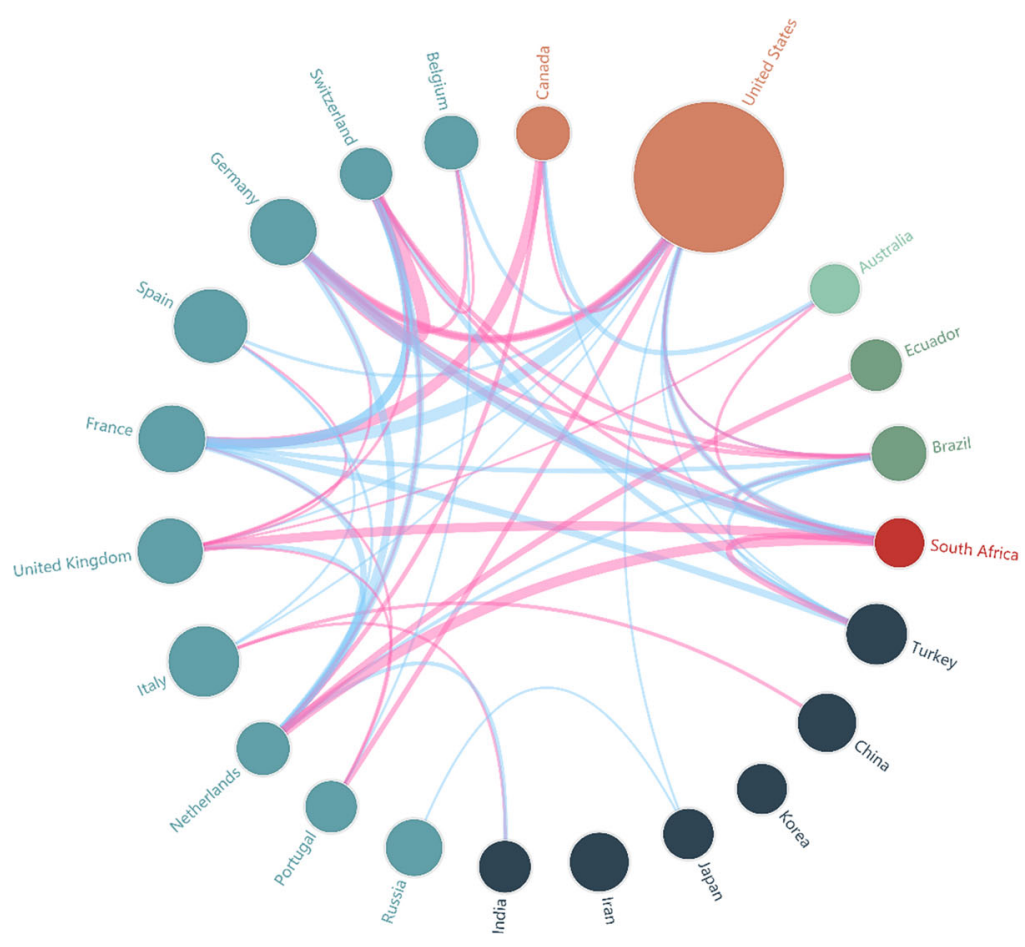

Fig. 2 Association between countries across six continents

or not on a specific day. The results in Table S4 show that the correlation patterns we observe are largely held unchanged and may simulate the situation of international travels with transitions in third countries. Finally, we replicate the analysis by using another COVID-19 dataset maintained by the Center for Systems Science and Engineering (CSSE) at Johns Hopkins University [17] which contains more sources of information from $\mathrm{CDC}$ and online media sources in many countries. Results show largely consistent pattern of the correlation on the COVID-19 epidemics across countries and continents, although country-specific impact coefficients vary in the global network. For instance, China is still found to be fully independent from the epidemics in all other countries. Also, the USA is found to be consistently correlated with the European countries regarding their respective epidemics. The results based on the data from Johns Hopkins University are presented in Tables S5 to S7, corresponding to three models with the international flight capacity, the number of international flights and without flight information. The significant associations presented in Table S5 are also illustrated in Figure S2.

Considering potential collinearities between the estimated coefficients, we conduct a stepwise approach that adds countries' variables continent by continent. Using the USA as an example, the stepwise analysis in Table S8 shows the consistent effects, that is, the correlations between other countries' epidemics and the daily new infections in the USA, remain largely same and robust regarding the effect sizes and significance levels. 


\section{Discussions}

By using the global human mobility data, this study provides the first comprehensive insights into the correlation between the epidemics across a number of countries with the introduction of international travel restrictions during the first 3 months of the COVID-19 pandemic. Specifically, although the epidemic started in Asia first, the study finds that the epidemics in Asian countries have diverse degrees of associations with countries in other continents. China, for example, was one of the first countries to face an outbreak and severely affected, but introducing international travel restriction targeting travelers from China is found to have little change on the correlation between the early epidemic in China and the outbreak later in other countries. In contrast, the lagged daily infection in Japan shows more correlations with other counties' epidemics even with the international travel restriction. The study further reveals intensive correlations between the epidemics across North American and European countries either before or after the banning of trans-Atlantic travel. Among many European countries, we also find a close circle of mutual correlations in different periods of COVID-19 epidemics.

The first important takeaway from the study is the importance of a holistic view of the COVID-19 transmission. Restrictions on nonessential international human mobility between countries are one critical strategy to fight against epidemic outbreaks [6]; however, little was known before regarding the proper ways to implement the strategy. As such, policymakers might focus only on the hotspot areas with a high transmission rate (which is not wrong) but overlook the pathway effect beyond the epidemic areas in the global network. Unfortunately, over the past 3 months, we have observed such a narrow perspective repeatedly occurring in many countries, focusing only on cutting off hotspot outbreak areas. New York Governor Andrew Cuomo recently start to question where the coronavirus that hit New York state came from: “...We closed the front door with the China travel ban, which was right...but we left the back door open because the virus had left China by the time we did the China travel ban" [21]. Through this study, we show that the establishment of a new holistic and networked view of pandemic transmission on a global scale is imperative, with the following important implications.

First of all, it cannot be simply assumed that the international travel ban from highrisk areas will be sufficient to control the epidemic once and for all while ignoring the dynamic spread of the epidemic in the global network. Policymakers in every country should keep abreast of population movements between countries and, through scientifically rigorous analysis, and foresee the pattern of epidemic transmission in the network. Accordingly, they should dynamically adjust the corresponding international restriction strategy promptly.

Second, the global perspective should also take into account the domestic movement restriction already implemented and its effects. Despite the early outbreak in Asian countries, their impact on other countries is not the same. Because of strict national and international restrictions imposed by China, the epidemic in China was developing in isolation: It did not affect other countries or was affected by other countries' epidemics. In contrast, the lack of strict and timely domestic restriction in some other countries, combined with their lack of global view on timely international restriction, contributed 
to the pandemic, which means these countries were subsequently affected by the epidemic in the second and third waves.

By the same token, as epidemics are gradually brought under control in the coming months, the effects and extent of national and international movement restriction should both be taken into account when countries decide to resume international economic and social activities. At the same time, it's also important to remain vigilant about the third-wave outbreak that may appear in Latin America and Africa. The holistic view should be applied promptly to guide the internal and international movement restriction policies in the areas.

Finally, our analysis also shows that there may exist factors outside the epidemic that are influencing countries' decisions on international travel restrictions. For instance, when the USA started to ban non-US citizens or permanent residents who had been in China in the past 14 days to enter the USA on February 2, 2020, there were 16 640 infected patients in China with the infection rate of 11.95 per million people. In comparison, when the USA banned the entry of European people on March 13, Italy itself had 17660 patients with an infection rate of 292.29 per million people. Therefore, this also adds another dimension of restriction timing that future research should be aware of.

As one of the first few studies to focus on a holistic view of the epidemic, the paper cannot avoid its limitations. First, our estimation model captures how potential infections spread as people move out of their destinations by multiplying the flight numbers and the number of cumulative infections in the destination countries. Since there is no reported infection number of China, either by WHO or JHU before January 22 , the potential spread values from China to any other countries remain zero. It means that the study cannot estimate the spread situation before January 22 and the findings of the study are limited to the situation after January 22. Since the flight dataset shows continuous outbound flights from China to other countries after 22 January (albeit with a decrease in number), potential infection transmission from China to other countries can still be well captured by the mobility data.

Second, all the restrictions were still in effect at the time of writing; therefore, we mainly focused on the short-term effects rather than a longitudinal examination. We recommend that scholars exercise caution when extrapolating our conclusions to longer periods. Third, some reliability concerns still exist related to the confirmed number reported and policy implementation; however, we could analyze only the available data. Furthermore, examining the restriction implementation process and efforts of the actions in a more detailed manner would increase the rigor and power of the initial analysis presented in this paper. Models that can predict the dynamic future is also the focus of the next steps.

\section{References}

[1] Forster, P., Forster, L., Renfrew, C., Forster, M.: Phylogenetic network analysis of Sars-Cov-2 genomes. Proc. Natl. Acad. Sci. 117(17), 9241-9243 (2020)

[2] Gonzalez-Reiche, A.S., Hernandez, M.M., Sullivan, M., Ciferri, B., Alshammary, H., Obla, A., Fabre, S., Kleiner, G., Polanco, J., Khan, Z., Alburquerque, B., van de Guchte, A., Dutta, J., Francoeur, N., Melo, B.S., Oussenko, I., Deikus, G., Soto, J., Sridhar, S.H., Wang, Y.-C., Twyman, K., Kasarskis, A., 
Altman, D.R., Smith, M., Sebra, R., Aberg, J., Krammer, F., Garcia-Sarstre, A., Luksza, M., Patel, G., Paniz-Mondolfi, A., Gitman, M., Sordillo, E.M., Simon, V., van Bakel, H.: Introductions and early spread of Sars-Cov-2 in the New York City area. medRxiv, p. 2020.2004.2008.20056929 (2020)

[3] Bavel, J.J.V., Baicker, K., Boggio, P.S., Capraro, V., Cichocka, A., Cikara, M., Crockett, M.J., Crum, A.J., Douglas, K.M., Druckman, J.N., Drury, J., Dube, O., Ellemers, N., Finkel, E.J., Fowler, J.H., Gelfand, M., Han, S., Haslam, S.A., Jetten, J., Kitayama, S., Mobbs, D., Napper, L.E., Packer, D.J., Pennycook, G., Peters, E., Petty, R.E., Rand, D.G., Reicher, S.D., Schnall, S., Shariff, A., Skitka, L.J., Smith, S.S., Sunstein, C.R., Tabri, N., Tucker, J.A., Linden, S.V.D., Lange, P.V., Weeden, K.A., Wohl, M.J.A., Zaki, J., Zion, S.R., Willer, R.: Using social and behavioural science to support COVID-19 pandemic response. Nat. Hum. Behav. 4, 460-471 (2020)

[4] West, R., Michie, S., Rubin, G.J., Amlôt, R.: Applying principles of behaviour change to reduce Sars-Cov-2 transmission. Nat. Hum. Behav. 4, 451-459 (2020)

[5] Wang, C., Liu, L., Hao, X., Guo, H., Wang, Q., Huang, J., He, N., Yu, H., Lin, X., Pan, A., Wei, S., and $\mathrm{Wu}, \mathrm{T}$.: Evolving epidemiology and impact of non-pharmaceutical interventions on the outbreak of coronavirus disease 2019 in Wuhan, China. medRxiv, p. 2020.2003.2003.20030593 (2020)

[6] Paules, C.I., Marston, H.D., Fauci, A.S.: Coronavirus Infections-more than just the common cold. JAMA 323, 707-708 (2020)

[7] Giordano, G., Blanchini, F., Bruno, R., Colaneri, P., Di Filippo, A., Di Matteo, A., Colaneri, M.: Modelling the Covid-19 epidemic and implementation of population-wide interventions in Italy. Nat. Med. 26, 855-860 (2020)

[8] Guan, W.-J., Ni, Z.-Y., Hu, Y., Liang, W.-H., Ou, C.-Q., He, J.-X., Liu, L., Shan, H., Lei, C.-L., Hui, D.S.C., Du, B., Li, L.-J., Zeng, G., Yuen, K.-Y., Chen, R.-C., Tang, C.-L., Wang, T., Chen, P.-Y., Xiang, J., Li, S.-Y., Wang, J.-L., Liang, Z.-J., Peng, Y.-X., Wei, L., Liu, Y., Hu, Y.-H., Peng, P., Wang, J.-M., Liu, J.-Y., Chen, Z., Li, G., Zheng, Z.-J., Qiu, S.-Q., Luo, J., Ye, C.-J., Zhu, S.-Y., Zhong, N.-S.: Clinical characteristics of coronavirus disease 2019 in China. N. Engl. J. Med. 382, 1708-1720 (2020)

[9] Huang, C., Wang, Y., Li, X., Ren, L., Zhao, J., Hu, Y., Zhang, L., Fan, G., Xu, J., Gu, X.: Clinical features of patients infected with 2019 novel coronavirus in Wuhan, China. Lancet 395, 497-506 (2020)

[10] Jia, J.S., Lu, X., Yuan, Y., Xu, G., Jia, J., Christakis, N.A.: Population flow drives spatio-temporal distribution of Covid-19 in China. Nature 582, 389-394 (2020)

[11] Kraemer, M.U.G., Yang, C.-H., Gutierrez, B., Wu, C.-H., Klein, B., Pigott, D.M., du Plessis, L., Faria, N.R., Li, R., Hanage, W.P., Brownstein, J.S., Layan, M., Vespignani, A., Tian, H., Dye, C., Pybus, O.G., Scarpino, S.V.: The effect of human mobility and control measures on the Covid-19 epidemic in China. Science 368, 493-497 (2020)

[12] Li, R., Pei, S., Chen, B., Song, Y., Zhang, T., Yang, W., Shaman, J.: Substantial undocumented infection facilitates the rapid dissemination of novel coronavirus (Sars-Cov2). Science 368, eabb3221 (2020)

[13] Wu, J.T., Leung, K., Leung, G.M.: Nowcasting and forecasting the potential domestic and international spread of the 2019-Ncov outbreak originating in Wuhan, China: a modelling study. Lancet (2020). https://doi.org/10.1016/S0140-6736(20)30260-9

[14] Lancet: Covid-19: too little, too late? Lancet 395, 10226 (2020)

[15] Belotti, F., Hughes, G., Piano Mortari, A.: Spatial panel data models using Stata. Stata J. 17(1), 139-180 (2017)

[16] Elhorst, J.P.: Spatial Econometrics: From Cross-Sectional Data to Spatial Panels. Springer, Heidelberg (2014)

[17] Dong, E., Du, H., Gardner, L.: An interactive web-based dashboard to track Covid-19 in real time. Lancet Infect. Dis. 20, 533-534 (2020)

[18] Ajmani, V.: Applied Econometrics Using the SAS System. Wiley, New York (2011)

[19] Greene, W.H.: Econometric Analysis. Prentice Hall, New Jersey (2003)

[20] Zellner, A.: An efficient method of estimating seemingly unrelated regressions and tests for aggregation bias. J. Am. Stat. Assoc. 57(298), 348-368 (1962)

[21] Schwartz, I.: Cuomo: The Coronavirus That Came to New York "Did Not Come from China, It Came from Europe" (2020). https://www.realclearpolitics.com/video/2020/04/24/cuomo_the_coronavirus_ that_came_to_new_york_did_not_come_from_china_it_came_from_europe.html. Accessed 26 Apr 2020 